\title{
Editorial: Doing Critical Health Communication: A Forum on Methods
}

\author{
Shaunak Sastry ${ }^{1 *}$, Heather M. Zoller ${ }^{1}$ and Ambar Basu ${ }^{2}$ \\ ${ }^{1}$ Department of Communication, University of Cincinnati, Cincinnati, $\mathrm{OH}$, United States, ${ }^{2}$ Department of Communication, \\ University of South Florida, Tampa, FL, United States
}

Keywords: health communication, critical health studies, research methods, reflexivity, critical health communication

\section{Editorial on the Research Topic}

"Doing" Critical Health Communication. A Forum on Methods

\section{OPEN ACCESS}

Edited and reviewed by: Rukhsana Ahmed, University at Albany, United States

${ }^{*}$ Correspondence: Shaunak Sastry sastrysk@ucmail.uc.edu

Specialty section: This article was submitted to Health Communication, a section of the journal Frontiers in Communication

Received: 03 December 2020 Accepted: 24 December 2020 Published: 25 January 2021

Citation:

Sastry S, Zoller HM and Basu A (2021) Editorial: Doing Critical Health Communication: A Forum on Methods. Front. Commun. 5:637579. doi: 10.3389/fcomm.2020.637579
The assumed premise of health communication research is straightforward: improving communication processes across all health-related domains. Communication between providers and patients, public health messaging, health literacy training, culturally competent healthcare, health status sharing in families, workplaces, and small groups can all fit within the broad definition of health communication. However, philosophical differences in what communication means-or for that matter, what health means-result in a complex, multi-paradigmatic field of study. For instance, viewing communication primarily as information transfer leads to a different trajectory of research and scholarship than a view of communication as the constitutive process of meaning making. Similarly, conceptualizing health as a means of achieving social concordance or even control vs. as a site of social struggle leads us different places.

Within the well-established field of health communication, a preponderance of published research continues to be rooted in communication models that derive from social psychology and information science. Consequently, emerging issues, new theoretical and methodological directions, and ethical challenges define the landscape of the field. For instance, we have witnessed a significant rise in interpretive research focusing on the social construction of meaning. However, we believe there is more work to do in nurturing critical health communication $[\mathrm{CHC}]$ perspectives.

The primary rationale for this research topic was to describe multiple ways to engage in $\mathrm{CHC}$ methodologies through a set of short, "how-to" articles. The original impetus were two roundtable panels (convened at successive National Communication Association conventions) to gauge the trajectory of CHC in the decade after Zoller and Kline's review of the contributions of interpretive/ critical health communication research in the Annals of Communication (then called Communication Yearbook). One of the things we recognized in those panel discussions was that $\mathrm{CHC}$ was still considered a niche sub-discipline or area within health communication, and consequently, students and young scholars who were interested in $\mathrm{CHC}$ often did not receive formal guidance in this area, notwithstanding the dramatic increases in CHC-fueled work being published in our disciplinary journals, and/or presented at conferences. Even for scholars familiar with the intellectual terrains of poststructuralism, postcolonialism, the "linguistic turn," hermeneutics, phenomenology and critical theory, there was a gap in documenting these theoretical concepts into concrete ways of "doing" health communication research.

In calling for papers, we urged potential authors to ask, "What makes your work critical?” How do methodological practices illuminate the role of critique? What are the ontological and epistemological implications of doing $\mathrm{CHC}$ ? How is $\mathrm{CHC}$ related to critical praxis? How does "doing" critical work engage with/deviate from the broader interpretive move toward discourses/ 
texts? What do recent provocations around the "return to the material" in Communication scholarship mean for $\mathrm{CHC}$ researchers? How is $\mathrm{CHC}$ situated to respond to widening racial, gendered and other social disparities in health across the globe? Finally, how do $\mathrm{CHC}$ researchers situate their own privilege and conceptualize embodied risk through their work? The fourteen articles that comprise this collection, selected from the $30+$ abstracts submitted for consideration, and shortlisted from 19 full-text article submissions) respond to this prompt in unique, individual ways.

Of the fourteen articles, five report on new/original research, four offer 'Conceptual Analysis' or brief essays on a particular concept. Another four are short "Perspectives" on varying issues concerning $\mathrm{CHC}$, and one is a Brief Research Report. As to our remit of a "how to" for $\mathrm{CHC}$, the articles offer pedagogical insights on $\mathrm{CHC}$ methods in a variety of ways.

Zoller and Kline's 2008 drew attention to both shared attributes and key points of difference in interpretive and critical health communication. One of our goals for this topic was to theorize their differences as well as their "blurry edges." Anne Kerber's essay addresses longstanding conflicts between a critical "hermeneutic" of suspicion that interrogates relations of power and an affirmative stance that seeks positive models of critical social change.

A second rationale for this collection was to re-establish the disciplinary history of the efforts of $\mathrm{CHC}$ scholars. At the abovementioned conference panel discussions and through our own anecdotal experience, we have learnt that the multi-decade project to critique, de-parochialize, globalize and queer the body of the discipline (and consequently, its journals and editorial boards), led by women, scholars of color, LGBTQ scholars, and scholars from the Global South, has not been documented or set into the received intellectual history of the field (in contrast to cognate areas, like critical organizational or critical management studies). This absence influences the diffusion of our work. It also makes it possible for other scholarly collectives, notably our colleagues who coalesce under the "Rhetoric of Health and Medicine" or RHM, whose work we admire, review and support, to largely ignore this history and the contributions of $\mathrm{CHC}$ scholars in opening up space for critical/humanist inquiry in this area. In that sense, we seek to make explicit the politics, the pragmatics and the real-life implications of doing CHC work. As a foundational scholar in the area, Heather Zoller's essay derives from her extensive work in the field, and outlines how the politics of academic training, visibility, and publishing intersect in pursuing a trajectory of critical health communication research. This essay is an excellent entry point for this research topic.

Essays in this collection model different forms of critical analysis. For instance, Carter and Alexander's original research is an exemplar for connecting race, class, historical positioning, and health communication practices. Their interview-based original research highlights the voices of African American farmers, revealing how their issues and interests have been silenced in discussions about United States farming. They connect these erasures with broader political discourses about diet and health disparities.
Khan et al. model critical ethnographic analysis through their study of Ashodaya Samithi, a sex worker collective in Mysore, India. They offer narratives that highlight resistance and alliance building that are imperative in order to invert dominant discriminatory notions of nationhood and citizenship that have and continue to violate health and rights of marginalized communities. Much of the critical work in health communication has emerged from the global South, espousing a critique of the West-dominated nature of communication theorizing and global health policies.

Dutta and his team provide a primer in a Marxist approach to critical theorizing, with attention to the global subaltern. The authors draw from their embodied culture-centered research engaging in activist interventions that aim to disrupt Whiteness and associated capitalist and colonial logics. The authors challenge us to consider what counts as resistance organizing in ways that provide an interesting counterpoint to Kerber's essay. Such tensions in what counts as "critical" research in health communication continues to be an important fault line in our field. Metatheoretical differences in conceptualizing the role of the critic in health communication manifest in methodological and pragmatic differences in what research looks like. One such difference is in the practice of what some scholas call 'critical reflexivity'

Critical reflexivity-or the continual introspection of how analysis reveals the motivations of the analyst as much as it says something about that which is analyzed-is a governing principle guiding the ethical conduct of critical research. Rebecca de Souza's essay interrogates how the literature on critical reflexivity-what she calls the "self-other" hyphen-predicates a white researcher introspecting on their ethical analytical practices as they work in communities of color. However, flipping the trope, de Souza's essay offers a fascinating look at what happens when a person of color navigates analysis of predominantly white spaces. Through an analysis of the responses and challenges to her work by peer reviewers, commentators and colleagues, de Souza offers a window into the "micro-politics" of knowledge production. Her work offers practical suggestions for scholars of color to challenge the hegemonic assumptions that emerge from working in white spaces.

Similarly, Leandra Hernandez and Sarah De Los Santos Upton provide an exemplar of the power of critical reflexivity and the need for critical praxis through social justice activism. The essay blends discussion of their research and activist work, describing the intersectional approach they have taken to health communication research at the United States-Mexico border. Situated as Chicana feminists, they have investigated gendered, racial and class constructions in the context of reproductive justice, violence, and immigration. The authors describe how their work has necessitated a blending of theoretical and methodological approaches.

Critical reflexivity is also an important tool in Smita Misra's essay, which centers around the concept of migrant trauma. As encapsulated by their experiences in a participatory theater project that purportedly allowed for refugees to cope with trauma, Misra offers a critical reflexive account of how well- 
meaning, "participatory"/critical projects can offer limited/ constraining understanding of the lives of the vulnerable populations they serve.

Nicole Hudak's essay discusses challenges in publishing research that does not fit within post-positivism, calling for more advocacy of qualitative and critical research. In addition, the essay challenges all of us to interrogate reviewer practices that reinforce heteronormativity and create barriers to research addressing LGBTQ + health care experiences. This turn to embodied identity is further crystallized in Ellingson's work, which theorizes embodiment more centrally.

Embodiment becomes sensorial in Laura Ellingson's essay. Sensual intersubjectivities that blend the senses, the motors, and the material, Ellingson explains, are crucial to critical health communication research methods because interrupting discourses on/of what makes certain bodies/citizens 'healthy' and 'normal' calls for a sustained practice of sensorial reflexivity.

If critical reflexivity is one way to redefine the "blurry edges" between interpretive and critical approaches, then Sastry and Basu's essay offers a methodological warrant to use critical reflexivity as a practicable method for analysis in health communication. The essay elucidates an approach blending culture-centered analysis, abductive analysis, and critical reflexivity in a post-COVID world. Departing from their ethnographic work in the culture-centered tradition, the authors offer a framework to analyze health discourses using the early responses to COVID-19 as an exemplar.

Several essays offer methodological innovations in the doing of critical health research. Sarah MacLean and Simon Hatcher write about the walkthrough method in their essay. The walkthrough method offers a viable process to scrutinize the architecture of a health technology tools -- the BEACON Rx Platform in their case -in terms of expected use and consequent implications of access and equity. This method also creates spaces for questioning the discourses inherent in health technologies that frame dominant understandings of how to be in "good" health.

Wendy Pringle provides a new methodological tool for critical health communication scholars, particularly those interested in textual/rhetorical analysis and policy discourses. She adapts the "What's the Problem Represented to be?" (WPR) approach from the field of discursive policy analysis. The paper uses the illustrative example of the legalization of medical assistance in dying in Canada. The WPR method facilitates attention to evolving discourses of problem constructions, and she describes the implications for people with disabilities, including what is said and what is left unspoken. The method addresses social change, including policy critique, and advocacy as a form of resistance.

In our call for papers, we hoped to collectively articulate (and complicate) what exactly we mean by "critical" in $\mathrm{CHC}$. In addition to the models we have discussed, Kim Kline and Shamshad Khan call attention to the need for CHC scholars to speak to both internal and external stakeholders. Their essay signposts the possibilities and challenges for $\mathrm{CHC}$ scholars to engage in "transdisciplinary" collaborations within and without the discipline of health communication.

Speaking of collaborations, this research topic would not have been realized without the collaborative efforts between the contributing authors, the editorial team, and most importantly, the large number of reviewers who volunteered their time and intellectual commitment to this cause-not to mention adapting their reviewing practices for Frontiers. While open-access, transparency, and publication of reviewers' names with published articles signals the timely democratization of the publication process, the concomitant "bot-tification" of the process was a learning curve for several Communication scholars-us included.

As we conclude this editorial, the United States has more than 13 million confirmed cases of COVID-19, and some estimates suggest that the death toll might reach $5,00,000$ by the summer of 2021. Debates around masks, vaccines, technology transfers, economic impacts and racial and income inequalities related to the pandemic continue, painfully demonstrate the need for more research in how mechanisms of power/control/inequality shape individual and collective experiences of health and illness.

\section{AUTHOR CONTRIBUTIONS}

All authors listed have made a substantial, direct, and intellectual contribution to the work and approved it for publication.

Conflict of Interest: The authors declare that the research was conducted in the absence of any commercial or financial relationships that could be construed as a potential conflict of interest.

Copyright (C) 2021 Sastry, Zoller and Basu. This is an open-access article distributed under the terms of the Creative Commons Attribution License (CC BY). The use, distribution or reproduction in other forums is permitted, provided the original author(s) and the copyright owner(s) are credited and that the original publication in this journal is cited, in accordance with accepted academic practice. No use, distribution or reproduction is permitted which does not comply with these terms. 